\title{
Atomic-Scale Carving of Nanopores into a van der Waals Heterostructure with Slow Highly Charged Ions
}

Janine Schwestka, ${ }^{* \dagger \dagger}$ Heena Inani, ${ }^{* \ddagger}$ Mukesh Tripathi, ${ }^{\ddagger}$ Anna Niggas, ${ }^{\dagger}$ Niall McEvoy, ${ }^{\circledR}$ Florian Libisch, $\stackrel{\S}{ }$ Friedrich Aumayr ${ }^{\dagger}$ Jani Kotakoski, ${ }^{\ddagger}$ and Richard A. Wilhelm ${ }^{\dagger, \|}$

$\dagger T U$ Wien, Institute of Applied Physics, Vienna, Austria $\ddagger$ University of Vienna, Faculty of Physics, Vienna, Austria ITrinity College Dublin, AMBER School of Chemistry, Dublin, Ireland $\S T U$ Wien, Institute for Theoretical Physics, Vienna, Austria

\|Helmholtz-Zentrum Dresden-Rossendorf, Institute of Ion Beam Physics and Materials Research, Dresden, Germany

E-mail: schwestka@iap.tuwien.ac.at; heena.inani@univie.ac.at;wilhelm@iap.tuwien.ac.at Supporting information

*These authors contributed equally to the work 


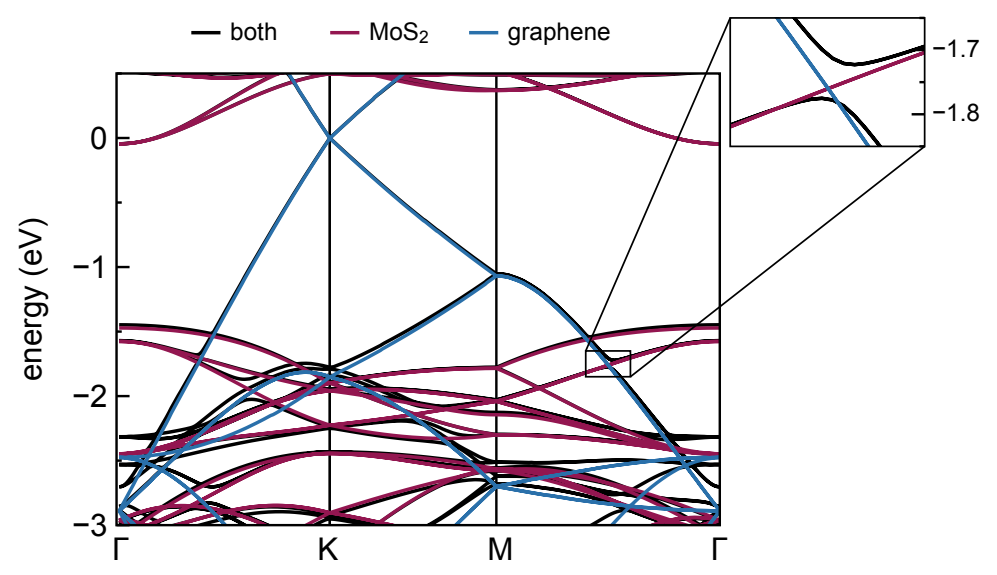

Figure 1: Band structure of a $3 \times 3 \mathrm{MoS}_{2}+4 \times 4$ graphene bilayer system from density functional theory (see methods). Band structures of the individual, separate layers are shown in red $\left(\mathrm{MoS}_{2}\right)$ and blue (graphene). Changes in the bandstructure are minimal, with small avoided crossings (see zoom-in).

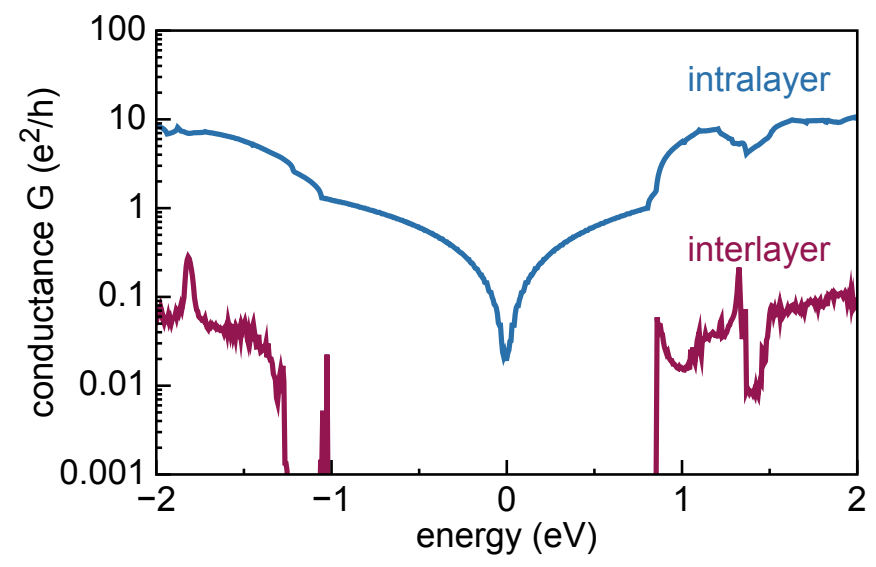

Figure 2: Intralayer conductance of graphene/ $\mathrm{MoS}_{2}$ heterostructure is at least two orders of magnitude larger than the interlayer conductance. 